\title{
Is the Dust Obscuring Supernovae in Distant Galaxies the Same as Dust in the Milky Way?
}

\section{Citation}

Riess, Adam G., William H. Press, and Robert P. Kirshner. 1996. "Is the Dust Obscuring Supernovae in Distant Galaxies the Same as Dust in the Milky Way?" The Astrophysical Journal 473 (2): 588-94. https://doi.org/10.1086/178174.

\section{Permanent link}

http://nrs.harvard.edu/urn-3:HUL.InstRepos:41417366

\section{Terms of Use}

This article was downloaded from Harvard University's DASH repository, and is made available under the terms and conditions applicable to Other Posted Material, as set forth at http:// nrs.harvard.edu/urn-3:HUL.InstRepos:dash.current.terms-of-use\#LAA

\section{Share Your Story}

The Harvard community has made this article openly available.

Please share how this access benefits you. Submit a story.

Accessibility 


\title{
IS THE DUST OBSCURING SUPERNOVAE IN DISTANT GALAXIES THE SAME AS DUST IN THE MILKY WAY?
}

\author{
Adam G. Riess, William H. Press, and Robert P. Kirshner \\ Harvard-Smithsonian Center for Astrophysics, 60 Garden Street, Cambridge, MA 02138 \\ Received 1996 April 15 ; accepted 1996 July 1
}

\begin{abstract}
Previous attempts to correct type Ia supernovae (SN Ia's) for host galaxy extinction have given strange results: increased dispersion on the Hubble diagram or impossibly low values of the reddening ratio for dust in distant galaxies. The cause is the incorrect assumption that SN Ia's have a uniform intrinsic luminosity and color at maximum light. Our multicolor light-curve shape (MLCS) method establishes the relation between intrinsic luminosity and color for SN Ia's using information in lightcurve shapes. Here we estimate the $B-V, V-R$, and $V-I$ color excess for $20 \mathrm{SN}$ Ia's using MLCS and estimate the reddening ratios of dust in distant galaxies. The ratios of selective to total absorption from dust in distant galaxies hosting SN Ia's are consistent with the galactic extinction law. The SN Ia's in late-type galaxies are often obscured by dust, while those in early-type galaxies are dust free. This suggests that SN Ia extinction is caused by interstellar (not circumstellar) dust, with similar optical properties as the dust in the Milky Way.
\end{abstract}

Subject headings: cosmology: observations - distance scale — dust, extinction — galaxies: ISM supernovae: general

\section{INTRODUCTION}

Previous attempts to measure the extinction of Type Ia supernovae (SN Ia's) have resulted in increased dispersion on the Hubble diagram and extreme properties for dust in distant galaxies. Sandage \& Tammann (1993) noted, "If the conventional correction for absorption in the blue of $A_{B} \sim$ $4 E_{B-V}$ is applied to each of the SN Ia's individually, the unacceptable consequence is that the scatter in the Hubble diagram is increased." Attempts to measure the ratio of $R_{B}=\left(A_{B} / E_{B-V}\right)$ for dust in distant galaxies from SN Ia's have given equally unacceptable results. We show here that the problem lies with past assumptions that SN Ia's were standard candles with a uniform luminosity and uniform intrinsic color at maximum light. We have shown elsewhere (Riess, Press, \& Kirshner 1996a, hereafter RPK 96a) that SN Ia's have intrinsic variations in color at maximum light that are correlated with variations in peak absolute magnitude. When these effects are understood, SN Ia's provide a way to evaluate the properties of interstellar dust in other galaxies.

One can solve easily for the reddening ratio, $R_{B}$, for the dust obscuring SN Ia's, if one assumes that SN Ia's at maximum have a uniform brightness in $B$ with a unique intrinsic color $(B-V)_{0}$. For two SN Ia's at the same distance, the difference in the apparent $B$ brightness divided by the difference in observed $B-V$ color gives $R_{B}$. For SN Ia's in the Hubble flow, the difference between the apparent brightness and the apparent brightness predicted by the redshift divided by the color excess gives $R_{B}$. The color excess is given by $E_{B-V}=\left(m_{B}-m_{V}\right)-(B-V)_{0}$. This latter method requires knowledge of SN Ia intrinsic color that in the past has often been taken as the color of the bluest (and presumably most reddened) SN Ia's observed.

The reddening ratio, $R_{B}$, has been well determined in our Galaxy by comparing the colors of reddened and unreddened stars of identical spectral type. With some variations at shorter wavelengths, the evidence suggests a remarkable homogeneity of the optical portion of the interstellar extinc- tion curve (IEC) in various parts of the Galaxy with $R_{B}=$ $4.1 \pm 0.15$ (Savage \& Mathis 1979; Whittet 1992; Mathis 1990). Calculations show that despite their nonstellar spectra, $\mathrm{SN}$ Ia's would possess a reddening ratio of $R_{B}=$ 4.2 in an environment with galactic dust (Buser 1978).

Previous analysis assuming that SN Ia's have a unique luminosity and color yield surprisingly small values for the reddening ratio. Using SN Ia's in the Virgo Cluster or pairs of SN Ia's in the same galaxy, Branch \& Tammann (1992) find $R_{B}=2.0$ and $R_{B}=1.2$, respectively, with limited data. Determination of $R_{B}$ on the Hubble diagram has resulted in $R_{B}=0.7 \pm 0.1$ (Branch \& Tammann 1992) and $R_{B}=1.3$ \pm 0.2 (Miller \& Branch 1990). Others have found values for $R_{B}$ ranging from 1 to 2 (Capaccioli et al. 1990, Jöeever 1982; Tammann 1987; Sandage \& Tammann 1993; Cadonau, Sandage, \& Tammann 1985). The largest measurement of the reddening ratio for supernovae in distant galaxies is $R_{B}=3.35 \pm 0.25$ (Della Valle \& Panagia 1992), and as we will show in $\S 4$, an updated estimate from their data set gives a somewhat lower result. Taken as a whole, the determination of $R_{B}$ from SN Ia's implies astonishingly different optical properties for dust in distant galaxies than in the Milky Way. A value of $R_{B}=1$ implies that $A_{V}=0$ for any value of $E_{B-V}$, while $R_{B}<1$ results in dust that makes light bluer. Such values for $R_{B}$ are difficult to reconcile with our current understanding of how dust affects light.

Measurements of absorption by dust with bright stars in nearby galaxies have given values for $R_{B}$ that are similar to the galactic value, with some exceptions. Bouchet et al. (1985) find $R_{B}=3.72 \pm 0.18$ and $R_{B}=44.1 \pm 0.15$ for the Small and Large Magellanic Clouds. Absorption by dust in M31 gives $3.5 \leq R_{B} \leq 5.3$, with variations linked to local metallicity (Walterbos 1986; Kron \& Mayall 1960; Hodge \& Kennicutt 1982; Iye \& Richter 1985). Brosch \& Loinger (1991) find $R_{B}=3.61 \pm 0.12$ from the dust lanes of NGC 7625, and Brosch (1988) finds $R_{B}=3.7$ for M104. A noteworthy exception is the determination of $R_{B}=2.4$ for NGC 2534 (Rifatto 1990). 
Branch \& Tammann (1992) summarized the possibilities why the reddening ratio for SN Ia's is so small: (1) the dust in spiral galaxies could be drastically different from the Galactic dust, (2) most of the extinction might be caused by circumstellar dust with very particular optical properties, (3) the observed spread of $(B-V)$ at maximum might be inflated by observational errors, or (4) the assumption of a unique intrinsic $(B-V)_{0}^{\max }$ for all SN Ia's could be invalid. They conclude, "The very small value of $\left[A_{V} / E_{B-V}\right]$ required by the available SN Ia data remains a mystery awaiting future resolution."

Recent work suggests that SN Ia's do not have a unique luminosity or color at maximum (Phillips 1993; Hamuy et al. 1995; Riess, Press, \& Kirshner 1995; RPK 96a). If the variations in intrinsic luminosity and color at maximum are related (i.e., correlated), then previous measurements of $R_{B}$ that rest on the standard SN Ia assumption are likely to be incorrect.

We have shown elsewhere that the intrinsic SN Ia luminosity and color at maximum are indeed related (RPK 96a). Our multicolor light-curve shape (MLCS) method estimates the intrinsic luminosity and color of a Type Ia supernova from the shapes of the observed light and color curves (RPK 96a). The correlation between light and color curve shape and intrinsic luminosity is derived first from a "training set" of supernovae with known distance and reddening estimates. The result of this training process is a one-parameter family of light curves and unreddened color curves that span the range of observed supernova light curves and intrinsic luminosity (see Fig. 1). Because dust has

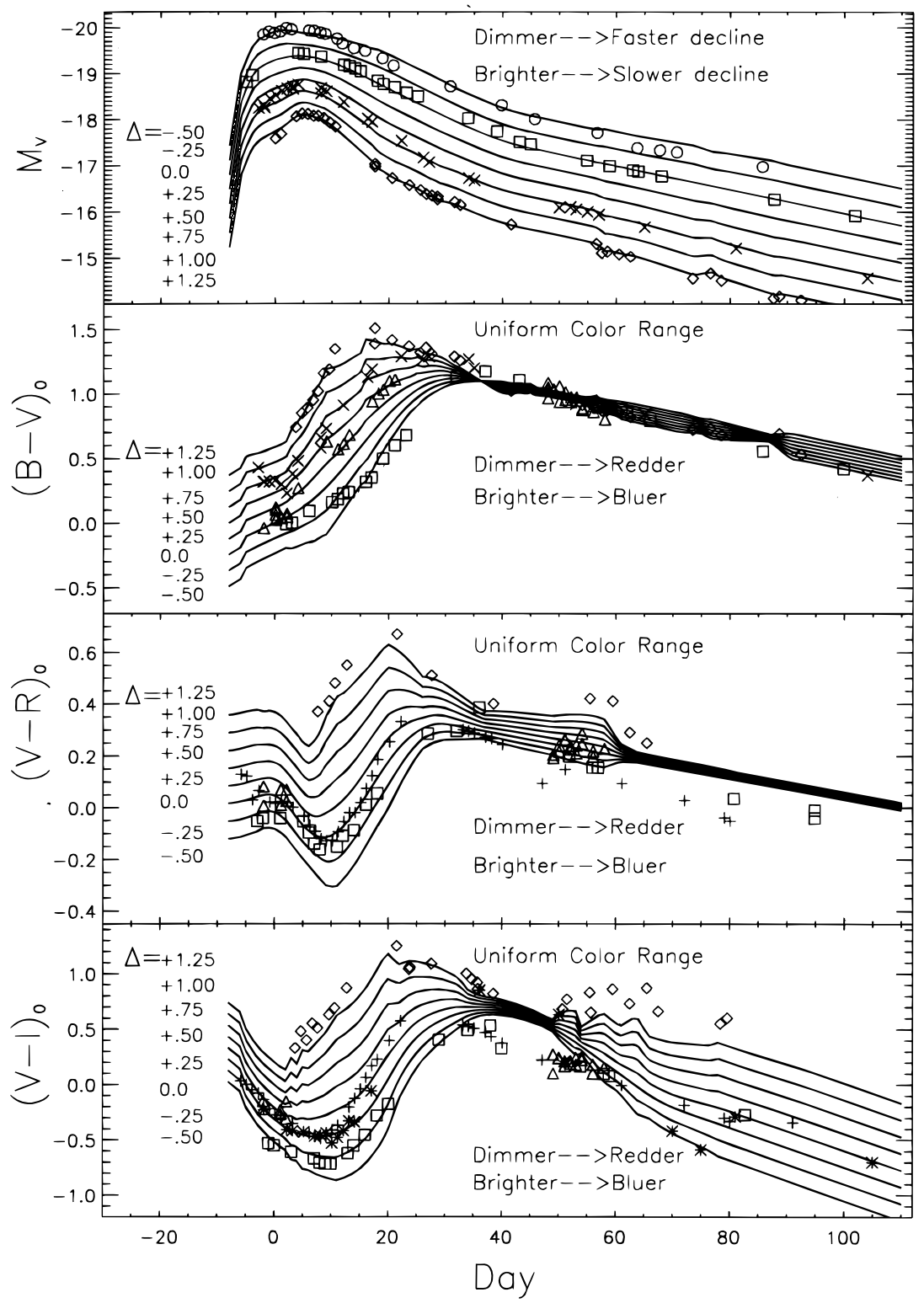

FIG. 1.-Empirical family of SN Ia light and color curves parameterized by luminosity. This sample of empirical $V, B-V, V-R$, and $V-I$ curves is derived from the training set and depicts the entire range of light and color curve shapes and their correlation with luminosity (on the Cepheid distance scale). This set is obtained by adding the correction templates, $R_{V}(t)$ or $R_{\text {color }}(t)$, multiplied by various luminosity corrections, $\Delta$, to the standard templates to make the best reconstruction of SN Ia light and color curves. From the multicolor light curve shape (MOCS) method, we estimate the luminosity and extinction by dust independently from the distance to measure the extinction-free distance. Data shown as reconstructed. Circles: 91T; squares: 94ae; crosses: 86G; damonds: $91 \mathrm{bg}$; plus signs: $92 \mathrm{~A}$; triangles: $80 \mathrm{~N}$. 
TABLE 1

SN Ia DATA

\begin{tabular}{|c|c|c|c|c|c|c|c|c|c|c|c|c|c|}
\hline $\begin{array}{l}\text { SN Ia } \\
\text { (1) }\end{array}$ & $\begin{array}{l}\log c z \\
\text { (2) }\end{array}$ & $\begin{array}{l}\mu_{v} \\
(3)\end{array}$ & $\begin{array}{l}\sigma_{\mu_{v}} \\
(4)\end{array}$ & $\begin{array}{c}\text { Galactic } \\
E_{B-V} \\
\text { (5) }\end{array}$ & $\begin{array}{c}E_{B-V} \\
\text { (6) }\end{array}$ & $\begin{array}{c}\sigma_{E_{B-V}} \\
(7)\end{array}$ & $\begin{array}{c}\sigma_{\mu_{V}, E_{B-V}}^{(8)} \\
\text { (8) }\end{array}$ & $\begin{array}{l}E_{V-R} \\
(9)\end{array}$ & $\begin{array}{l}\sigma_{E_{V-R}} \\
(10)\end{array}$ & $\begin{array}{c}\sigma_{\mu_{V}, E_{V}-R} \\
(11)\end{array}$ & $\begin{array}{c}E_{V-I} \\
(12)\end{array}$ & $\begin{array}{c}\sigma_{E_{V-I}} \\
(13)\end{array}$ & $\begin{array}{c}\sigma_{\mu_{V}, E_{V}-I} \\
\text { (14) }\end{array}$ \\
\hline 1992bo....... & 3.734 & 34.597 & 0.076 & 0.000 & 0.000 & 0.007 & $8.0 \mathrm{E}-05$ & 0.000 & 0.003 & $1.7 \mathrm{E}-05$ & 0.000 & 0.003 & $6.4 \mathrm{E}-05$ \\
\hline $1992 b c \ldots \ldots$ & 3.779 & 34.755 & 0.050 & 0.000 & 0.000 & 0.010 & $5.3 \mathrm{E}-05$ & 0.000 & 0.004 & $1.3 \mathrm{E}-05$ & 0.000 & 0.006 & $7.1 \mathrm{E}-05$ \\
\hline $1992 \mathrm{~K} \ldots \ldots$ & 3.521 & 3.548 & 0.157 & 0.075 & 0.041 & 0.024 & $2.7 \mathrm{E}-05$ & $\ldots$ & $\ldots$ & $\ldots$ & 0.000 & 0.046 & $5.5 \mathrm{E}-04$ \\
\hline 1992aq ....... & 4.481 & 38.269 & 0.064 & 0.000 & 0.000 & 0.010 & $8.0 \mathrm{E}-05$ & $\ldots$ & $\ldots$ & $\ldots$ & 0.000 & 0.006 & $3.8 \mathrm{E}-05$ \\
\hline 1992ae ....... & 4.350 & 37.795 & 0.061 & 0.015 & 0.000 & 0.026 & $9.2 \mathrm{E}-05$ & $\ldots$ & $\ldots$ & & & & \\
\hline $1992 P \ldots \ldots$ & 3.896 & 35.612 & 0.041 & 0.000 & 0.006 & 0.023 & $3.1 \mathrm{E}-05$ & $\ldots$ & 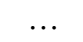 & $\ldots$ & 0.101 & 0.052 & $1.8 \mathrm{E}-05$ \\
\hline 1992J .. & 4.140 & 36.948 & 0.108 & 0.045 & 0.148 & 0.064 & $1.5 \mathrm{E}-04$ & & $\ldots$ & $\ldots$ & 0.012 & 0.142 & $2.5 \mathrm{E}-03$ \\
\hline 1991U ....... & 3.991 & 36.423 & 0.176 & 0.060 & 0.244 & 0.027 & $8.9 \mathrm{E}-05$ & 0.096 & 0.027 & $1.9 \mathrm{E}-04$ & 0.510 & 0.065 & $1.5 \mathrm{E}-03$ \\
\hline 1991ag . & 3.613 & 34.161 & 0.085 & 0.045 & 0.000 & 0.012 & $5.5 \mathrm{E}-04$ & 0.024 & 0.021 & $-1.3 \mathrm{E}-05$ & 0.132 & 0.059 & $2.4 \mathrm{E}-04$ \\
\hline 1990af . & 4.178 & 36.848 & 0.071 & 0.015 & 0.000 & 0.005 & $5.8 \mathrm{E}-05$ & & & & & & \\
\hline $1992 \mathrm{G}$. & 3.299 & 32 & 0.089 & 0.015 & & 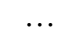 & & 0.006 & 0.018 & $2.8 \mathrm{E}-05$ & 0.307 & 0.053 & $4.4 \mathrm{E}-04$ \\
\hline $1991 \mathrm{~m}$. & 3.389 & 32.964 & 0.110 & 0.000 & & $\ldots$ & $\ldots$ & 0.000 & 0.014 & $4.1 \mathrm{E}-05$ & 0.000 & 0.020 & $2.1 \mathrm{E}-04$ \\
\hline 1993ae . & 3.736 & 34.531 & 0.135 & 0.030 & 0.000 & 0.014 & $5.9 \mathrm{E}-05$ & 0.000 & 0.008 & $7.9 \mathrm{E}-05$ & 0.000 & 0.035 & $9.3 \mathrm{E}-04$ \\
\hline 1994M . & 3.859 & 35.324 & 100 & 0.000 & 0.013 & 0.035 & $2.6 \mathrm{E}-04$ & 0.000 & 0.016 & $7.1 \mathrm{E}-05$ & 0.000 & 0.012 & $9.3 \mathrm{E}-05$ \\
\hline 1994S .. & 3.685 & 34.246 & 0.063 & 0.000 & 0.000 & 0.017 & $2.3 \mathrm{E}-05$ & 0.000 & 0.017 & $7.1 \mathrm{E}-05$ & 0.000 & 0.011 & $5.9 \mathrm{E}-05$ \\
\hline $1994 \mathrm{~T}$. & 4.030 & 36.165 & 0.114 & 0.000 & 0.000 & 0.017 & $1.4 \mathrm{E}-04$ & 0.021 & 0.055 & $6.9 \mathrm{E}-04$ & 0.000 & 0.023 & $5.3 \mathrm{E}-04$ \\
\hline 1994Q . & 3.935 & 36.117 & 0.138 & 0.015 & 0.027 & 0.032 & $1.4 \mathrm{E}-04$ & 0.119 & 0.018 & $8.0 \mathrm{E}-05$ & 0.103 & 0.054 & $6.9 \mathrm{E}-04$ \\
\hline 1993ac. & 4.170 & 37.12 & 239 & 0.150 & 0.002 & 0.032 & $3.2 \mathrm{E}-04$ & 0.014 & 0.060 & $4.0 \mathrm{E}-03$ & 0.133 & 0.102 & $6.2 \mathrm{E}-03$ \\
\hline 1995D ....... & 3.415 & 33.070 & 0.052 & 0.000 & 0.033 & 0.013 & $9.4 \mathrm{E}-06$ & 0.032 & 0.014 & $7.2 \mathrm{E}-06$ & 0.162 & 0.038 & $7.8 \mathrm{E}-05$ \\
\hline 1995E ........ & 3.539 & 35.516 & 0.043 & 0.000 & 0.738 & 0.025 & $1.0 \mathrm{E}-05$ & 0.467 & 0.023 & $1.0 \mathrm{E}-05$ & 0.928 & 0.030 & $2.1 \mathrm{E}-05$ \\
\hline
\end{tabular}

a Burstein \& Heiles 1982.

little effect on the observed shapes of light and color curves, the shapes can be used to estimate the SN Ia intrinsic luminosity and colors. The color excess is the decrease in the observed color curve needed to fit the intrinsic color curve shape for a supernova of that luminosity.

Our approach differs markedly from previous measurements of $R_{B}$ that assumed a unique intrinsic color and luminosity to measure the observed color excess and absorption (Branch \& Tammann 1992; Miller \& Branch 1990;
Capaccioli et al. 1990; Jöeever 1982; Tammann 1987; Sandage \& Tammann 1993; Della Valle \& Panagia 1992; Cadonau et al. 1985). We estimate the intrinsic luminosity and color for each SN Ia from the shape of its observed light and color curves. Accounting for the individual variation of intrinsic color and luminosity provides a more reliable estimate for the reddening ratios. Our rich and precise data set of CCD-based BVRI light curves allows us to measure two reddening ratios unavailable to previous
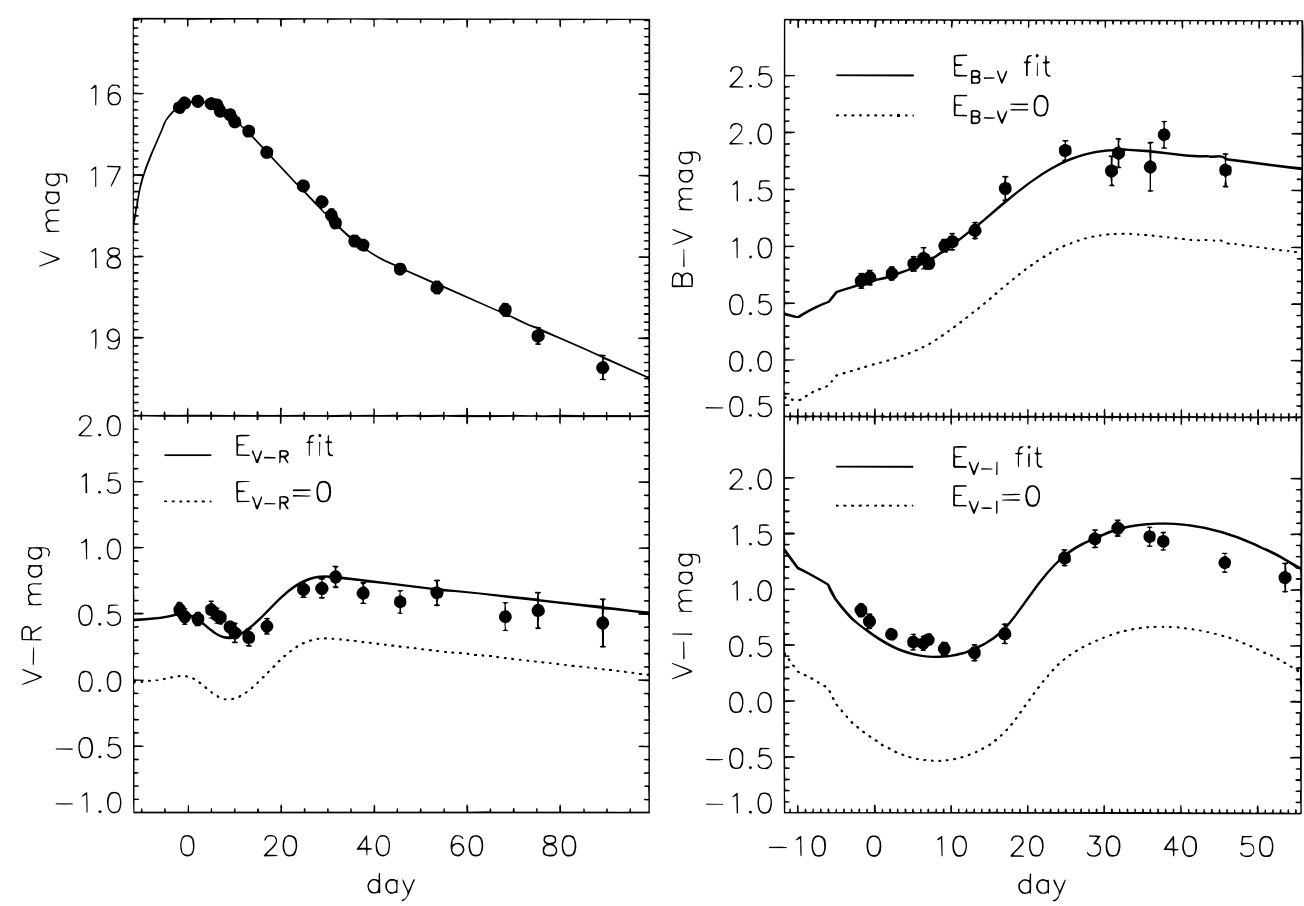

FIG. 2.-Example SN Ia MLCS fit to $V, B-V, V-R$, and $V-I$ data. Using the empirical light and color curve luminosity families in Fig. 1, we have fitted the shapes of the observed curves to the best matching intrinsic curves. This accounts for intrinsic variations in luminosity and color in the determination of the distance and color excesses. The distance modulus (uncorrected for extinction) is determined from the $V$ light-curve fit. Each color excess is determined independently by the redward displacement of the observed color curve from the best-fitting unreddened color curve. For SN $1995 \mathrm{E}$, the estimated color excesses are $0.74 \pm 0.03,0.47 \pm 0.02$, and $0.93 \pm 0.03$ for $E_{B-V}, E_{V-R}$, and $E_{V-I}$, respectively. 
authors with only $B$ and $V$ data. With three reddening ratios, we can compare the optical portion of the mean extinction curve of the distant host galaxies to that of the Milky Way.

In $\S 2$ we apply the MLCS method to an independent data set of 20 SN Ia's. We show in $\S 3$ that the results favor the hypothesis that the mean reddening properties of dust in distant galaxies are similar to those of galactic dust. We show also that the observed relation between host galaxy absorption along the line of sight to SN Ia and galaxy morphology is consistent with the standard picture of the dust content of galaxies. In $\S 4$ we demonstrate that the cause of the previous low measurements of $R_{B}$ from SN Ia's was inadequate understanding of the relation between intrinsic color and luminosity at maximum.

\section{DATA SET}

For 20 SN Ia's we have solved for the color excesses and their errors for up to three colors (as available); $B-V$, $V-R$, and $V-I$ (see Table 1). The fitting procedure is described in detail in RPK 96a. An example of this fitting procedure is seen in Figure 2. The photometry for the first 10 supernovae (col. [1]) comes from the Calán/Tololo Survey (Hamuy et al. 1993, 1994, 1995; Maza et al. 1994); SN 1991M and SN 1992G are from Ford et al. (1993), and the last eight are from our own work (Riess et al. 1996b). The redshifts (col. [2]) are from the same sources as the photometry and have been converted to the cosmic microwave background frame (see RPK 96a). The distances (uncorrected for extinction) (col. [3]) and their errors (col. [4]) come from the visual band light-curve shapes (RPK 96a). The distance scale rests on the Hubble Space Telescope Cepheid calibration of SN 1972E in NGC 5253, SN 1981B in NGC 4536, and SN 1990N in NGC 4639 (Sandage et al. 1994, 1996) and gives $H_{0}=65 \pm 6 \mathrm{~km} \mathrm{~s}^{-1} \mathrm{Mpc}^{-1}$ (RPK 96a). The calibration of the SN Ia luminosity and the determination of the distance scale has little effect on the reddening ratios, which result from magnitude differences.

The color excess (cols. [6], [9], and [12]) in $B-V, V-R$, and $V-I$ is measured as the redward displacement of the observed color curve from the best-fitting unreddened color curve. For a given SN Ia, each color excess is fitted independently. These values give the total line-of-sight reddenings to each SN Ia. In column (5) we give the Burstein \& Heiles (1982) estimate of the $B-V$ color excess due to galactic absorption in the direction of each SN Ia. In the following analysis, we have subtracted this component of galactic absorption from our measures of color excess and distance using the galactic ratios listed in Table 2. The standard errors in color excess (cols. [7], [10], and [13]) as well as the covariance between the color excess and distance determination (cols. [8], [11], and [14]) are given.

\section{ANALYSIS AND RESULTS}

There are two ways we can use the SN Ia data in Table 1 to compare the reddening properties of dust in distant gal- axies to those of galactic dust. One is to measure the ratio of different color excesses for the same SN Ia. The other is to measure the reddening ratios for distant galaxies by minimizing the component of Hubble law dispersion attributable to absorption.

We will make a maximum-likelihood analysis of three reddening ratios for the three colors using as a statistic the $\chi^{2}$ of the fit to the Hubble diagram. Using the definition that $R_{V \text {, color }}=\left(A_{V} / E_{\text {color }}\right)$, where color $=B-V, V-R$, or $V-I$, the extinction-corrected distance is given by $\mu_{V}-R_{V \text {,color }} E_{\text {color }}$. The most likely value of $R_{V \text {, color }}$ is that which minimizes the difference between $\sum_{i=1}^{N} \log c z_{i}$ and $\sum_{i=1}^{N} 0.2\left(\mu_{V, i}-R_{V, \text { color }} E_{\text {color }, i}\right)+\log H_{0}-5$ normalized by the errors. The $\chi^{2}$ of the Hubble line fit is given by

$$
\begin{aligned}
& \chi^{2}\left(H_{0}, R_{V, \text { color }}\right)= \\
& \sum_{i=1}^{N} \frac{\left[\log c z_{i}-0.2\left(\mu_{V, i}-R_{V, \text { color }} E_{\text {color }, i}\right)-\log H_{0}+5\right]^{2}}{\sigma_{i}^{2}},
\end{aligned}
$$

where

$$
\sigma_{i}^{2}=\sigma_{\mu_{V}, i}^{2}+\left(R_{V, \text { color }} \sigma_{E_{\text {color }, i}}\right)^{2}-2 R_{V, \text { color }} \sigma_{\mu_{V}, E_{\text {color }, i}} .
$$

Della Valle \& Panagia (1992) noted the importance of considering uncertainties in both the independent (distance) and dependent (color excess) variables. Neglecting uncertainties in color excess estimates is a part of the reason for historically low values for the reddening ratios from SN Ia's (see § 4).

Minimizing $\chi^{2}$ with respect to $\log H_{0}$ and $R_{V \text {,color }}$ determines the best values. The value of $\log H_{0}$ is linear in the equation $\left(\partial \chi^{2} / \partial \log H_{0}\right)=0$ and yields

$\log H_{0}=\frac{\sum_{i=1}^{N} \sigma_{i}^{-2}\left[\log c z_{i}-0.2\left(\mu_{V, i}-R_{V, \text { color }} E_{\mathrm{color}, i}\right)+5\right]}{\sum_{i=1}^{N} \sigma_{i}^{-2}}$.

The occurrence of the reddening ratios, $R_{V \text {, color }}$, in equation (2) requires a numerical determination of its best value and error. In the summations, we include all SN Ia's with color data and whose color excess (after removing galactic absorption) is nonnegative. For the colors $B-V$, $V-R$, and $V-I$ the number of suitable SN Ia's is 12,9 , and 13 , respectively. The best values for the reddening ratios are given in Table 2. The fits to the data give $\chi^{2}$ per degree of freedom of $1.20,0.27$, and 1.10 (probability of exceeding $\chi^{2}$ by chance is $0.29,0.96$, and 0.36 ) and are plotted in Figure 3 . The values for the reddening ratios in the Galaxy, determined from reddened and unreddened stars, are from Savage \& Mathis (1979) and are listed in Table 2. For each of the three colors, the extinction corrections reduce the dispersion from $\sim 0.6 \mathrm{mag}$ to $\sim 0.2 \mathrm{mag}$. In practice, the best way to correct the SN Ia luminosity for extinction is to combine all three color excesses (RPK 96a).

The values of $A_{V} / E_{B-V}$ from SN Ia's in distant galaxies is not inconsistent with the canonical galactic value and is well within the range observed in nearby galaxies (see $\S 1$ ). It

TABLE 2

REDDENING RATIOS

\begin{tabular}{cccccc}
\hline \hline Source & $A_{V} / E_{B-V}$ & $A_{V} / E_{V-R}$ & $A_{V} / E_{V-I}$ & $E_{V-I} / E_{V-R}$ & $E_{B-V} / E_{V-R}$ \\
\hline Galactic ..... & $3.10 \pm 0.15$ & $3.90 \pm 0.20$ & $1.90 \pm 0.10$ & $2.05 \pm 0.10$ & $1.28 \pm 0.05$ \\
SN Ia ....... & $2.55 \pm 0.30$ & $4.15 \pm 0.25$ & $1.95 \pm 0.20$ & $2.05 \pm 0.20$ & $1.55 \pm 0.15$ \\
\hline
\end{tabular}



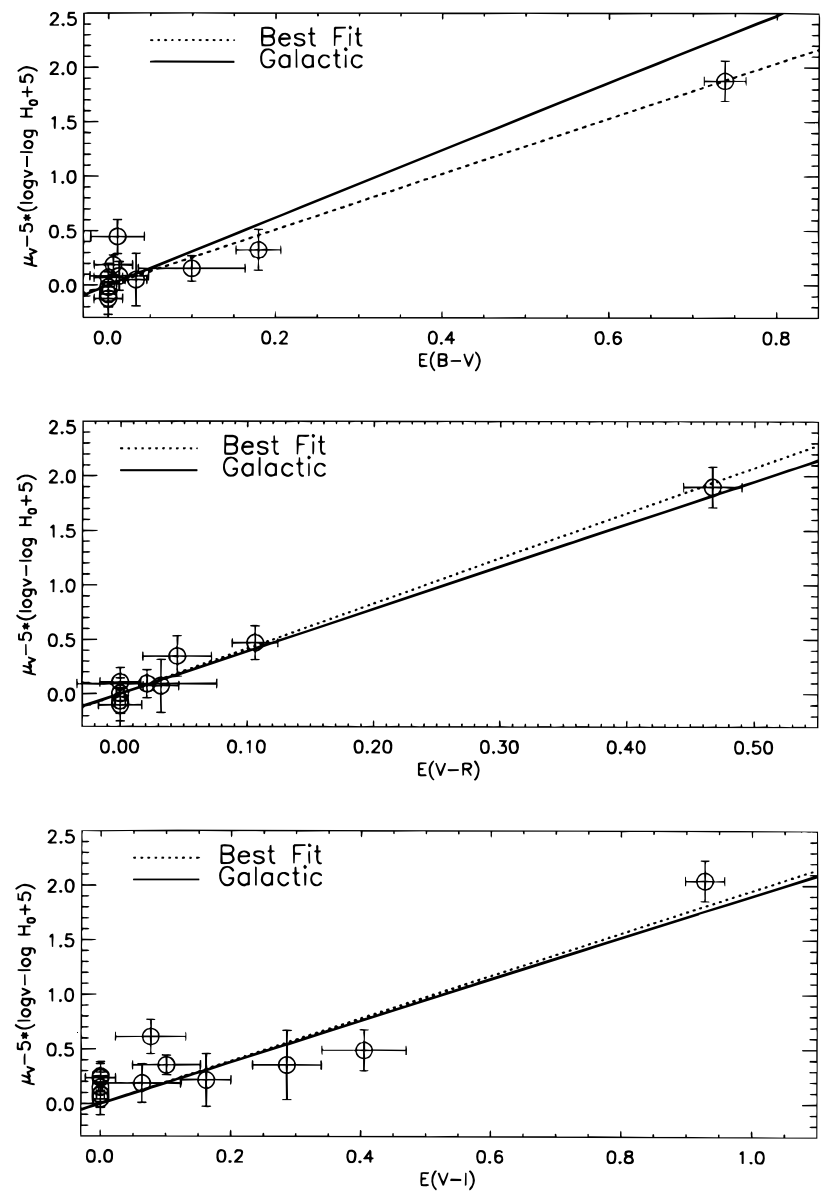

FIG. 3.-Maximum-likelihood estimates of three reddening ratios using the fit to the Hubble line as a $\chi^{2}$ statistic. We determine $A_{V} / E_{B-V}$, $A_{V} / E_{V-R}$, and $A_{V} / E_{V-I}$ by minimizing the component of Hubble law dispersion attributable to absorption from dust in distant galaxies. Estimates for the reddening ratios (dotted lines) are shown along with the canonical galactic values (solid line) (Savage \& Mathis 1979).

is also compatible with the variations in the $B$-band reddening ratio seen along various galactic lines of sight (Mathis 1990). Our value is significantly higher than the surprisingly low values of $-0.3 \leq\left(A_{B} / E_{B-V}\right) \leq 1.4$ found by others using SN Ia's (Branch \& Tammann 1992; Miller \& Branch 1990; Capaccioli et al. 1990; Jöeever 1982; Tammann 1987; Sandage \& Tammann 1993). [Note that $\left(A_{B} / E_{B-V}\right)-1=\left(A_{V} / E_{B-V}\right)$.] The values of $A_{V} / E_{V-R}$ and $A_{V} / E_{V-I}$ for dust in distant galaxies are highly consistent with the galactic values. The comparison between the reddening properties of galactic dust and dust in distant galaxies can be seen best in a plot of the reddening ratios from SN Ia's and the galactic interstellar extinction curve (see Fig. 4). The reddening properties of dust-obscuring SN Ia's are remarkably consistent with typical galactic absorption.

A powerful way to quantify the reddening effect of dust in distant galaxies independent of the Hubble law is to measure the ratio of different color excesses. For the SN Ia's, there are two unique ratios: $E_{V-I} / E_{V-R}$, and $E_{B-V} / E_{V-R}$ (see Fig. 5). To determine each ratio, we have used all SN Ia's that have data for both colors and for which both color excesses (after removing galactic absorption) are nonnegative. There were nine suitable SN Ia's for the two ratios $E_{V-I} / E_{V-R}$ and $E_{B-V} / E_{V-R}$. The

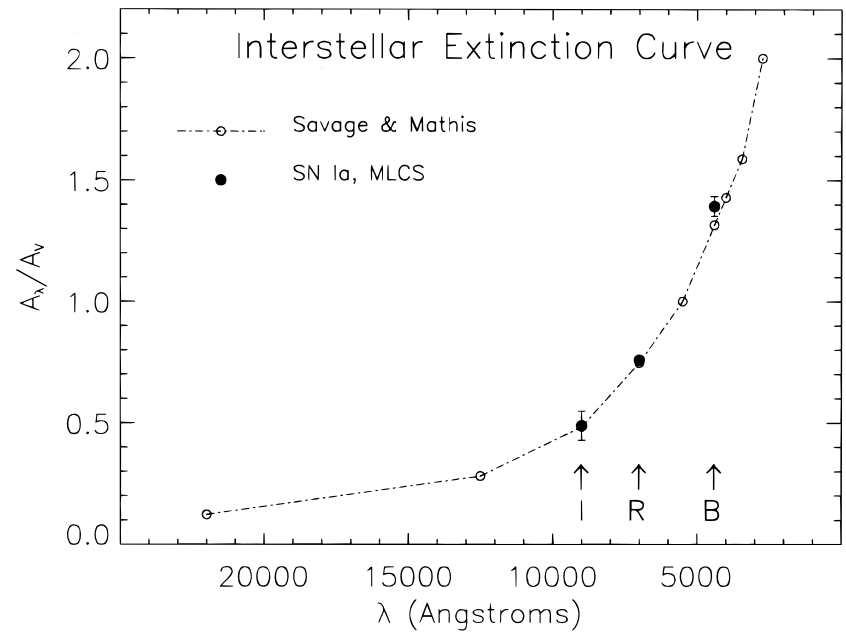

FIG. 4.-Comparison of the galactic interstellar extinction curve with three reddening ratios determined from the dust obscuring SN Ia's in distant galaxies. Taken as whole, the mean reddening properties of dust in distant galaxies are consistent with reddening by galactic dust.

best values for these ratios from the SN Ia's are given in Table 2 along with the values for the Galaxy (Savage \& Mathis 1979). The $E_{V-I} / E_{V-R}$ ratio from SN Ia's in distant galaxies is highly consistent with the value from galactic dust. The other ratio, $E_{B-V} / E_{V-R}$, is not inconsistent with the galactic values. This ratio samples the $B$-band absorption where spatial variations in the reddening law are observed in our Galaxy (Mathis 1990).

Besides refining our distance measurements, the MLCS estimates of the extinction from dust provide useful probes of the relation between galaxies and dust. In Figure 6 we explore the dependence of host galaxy type on measured extinction by dust. By removing the small Galactic component of obscuration (Burstein \& Heiles 1982) from the total $A_{V}$ as determined by MLCS (see Table 3 of RPK 96a),
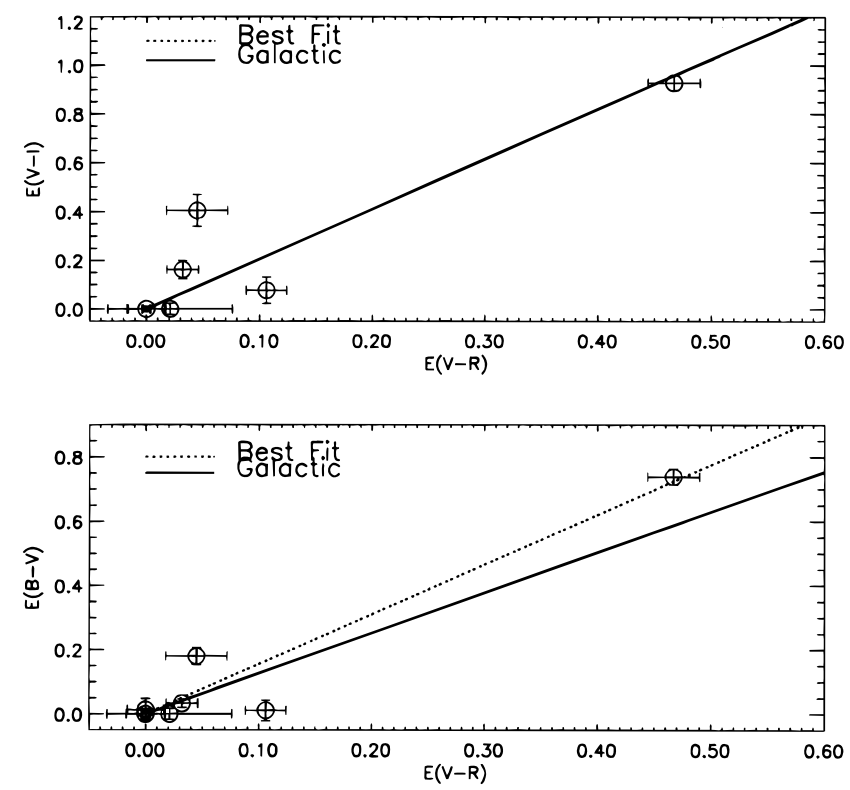

FIG. 5.-Measured ratio of color excesses from SN Ia's and the expectation for galactic dust. The two plotted ratios (dotted line) of color excesses are generally consistent with the ratios expected from galactic dust (solid line), but the results are strongly dependent on the highly reddened SN $1995 \mathrm{E}$. 


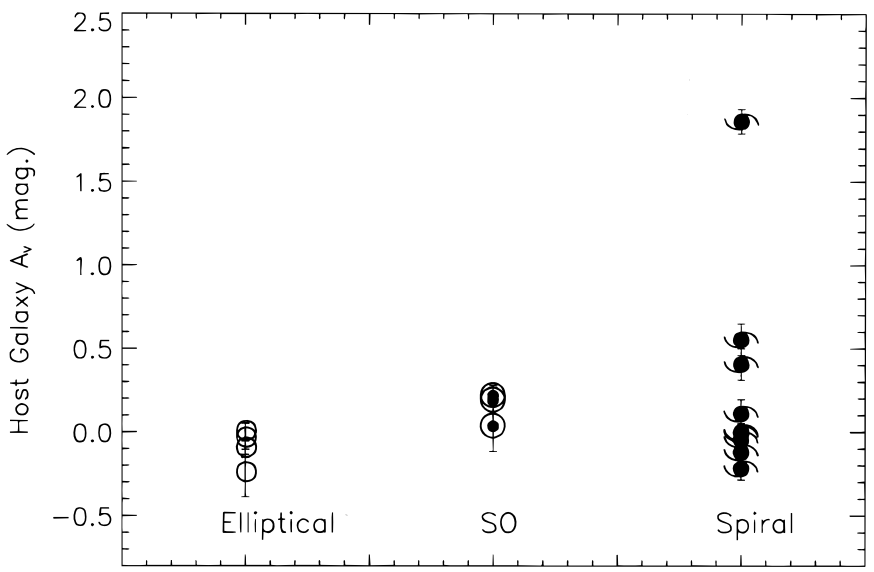

Galaxy Morphology

FIG. 6.-Relationship between the host galaxy extinction of SN Ia's and host galaxy morphology. The result is consistent with the standard picture of early-type galaxies being dust poor and late-type galaxies being dust rich. This suggests that the origin of SN Ia extinction is interstellar and not circumstellar dust.

we measure the obscuration from the host galaxy along our line of sight. We find that the extinctions measured to supernovae in elliptical galaxies are all consistent with zero. In S0 galaxies, we see a small range of nontrivial extinctions between 0.2 and $0 \mathrm{mag}$ of $A_{V}$. This range increases for supernovae in spiral galaxies, as seen in the highly obscured SN 1995E, whose visual brightness is dimmed by 2 mag of dust in the spiral arms of NGC 2441.

\section{DISCUSSION}

Our multicolor light-curve shape method provides a way to compare the reddening properties of dust in our Galaxy to those in distant galaxies. Accounting for the intrinsic variation of luminosity and color suggests that the mean absorption characteristics of dust in distant galaxies are consistent with those of dust in our Galaxy.

The strength of our conclusions depends strongly on the heavily reddened SN 1995E. Because of the selection bias against finding such objects, the sample of highly extinguished SN Ia's in the Hubble flow is severely limited. Until more of these useful SN Ia's are observed, the analysis of the dust obscuring SN Ia's will be limited by the small number of observed SN Ia's with significant extinction. In the future, we hope that astronomers will assume the challenge of observing highly extinguished SN Ia's because these objects are so useful in evaluating the properties of dust in distant galaxies.

Previous attempts to measure the reddening ratio, $A_{V} / E_{B-V}$, from SN Ia's in distant galaxies have, as a whole, yielded values significantly smaller than what is observed in our Galaxy or nearby galaxies (Branch \& Tammann 1992; Miller \& Branch 1990; Capaccioli et al. 1990; Jöeever 1982; Tammann 1987; Sandage \& Tammann 1993; Cadonau et al. 1985).

The only apparent exception, Della Valle \& Panagia's (1992) value of $\left(A_{V} / E_{B-V}\right)=2.35 \pm 0.25$, gives, upon closer scrutiny, a somewhat lower value for the reddening ratio. Assuming SN Ia's have a unique luminosity and color at maximum, these authors perform a maximum-likelihood analysis to determine the reddening ratio from 23 primarily photographic light curves. The authors include uncertainties in the independent and dependent variables but have underestimated these uncertainties, as reflected in their $\chi^{2}$ per degree of freedom, which is significantly greater than 1.0. Revised estimates for distance and color uncertainties for SN Ia's treated as standard candles have been provided by N. Panagia (1996, private communication). When we analyze their data with these error estimates, we find $\left(A_{V} / E_{B-V}\right)=1.7-2.2$. This value for the reddening ratio is significantly larger than previous values cited in $\S 1$ and results from including independent and dependent variable uncertainty. It is, however, significantly smaller than the value of $\left(A_{V} / E_{B-V}\right)=3.1 \pm 0.15$ from interstellar dust in the Galaxy.

Returning to Branch \& Tammann's (1992) summary of possibilities why the reddening ratio for SN Ia's is so small (see $\S 1$ ), our results from the set of 20 distant SN Ia's allow us to reject options (1), (2), and (3). The reddening ratios and excess color ratios we have derived show that, in the mean, the optical properties of dust in distant galaxies are not drastically different from those of galactic dust. Our results, however, do not preclude the possibility that the dust in individual galaxies may have different reddening properties than galactic dust. In particular, a close inspection of Figure 5 suggests there may be galaxies whose dust does not conform to the mean behavior. Figure 6 suggests strongly that the interstellar environment and not the circumstellar environment is the source of absorption by dust. If circumstellar dust were responsible for most of the SN Ia absorption seen, we would expect the measured $A_{V}$ to be independent of galaxy morphology, which it is clearly not. It is also difficult to blame the low values of $A_{V} / E_{B-V}$ on large errors in measured $(B-V)$ at maximum. Significant noise in the $(B-V)$ measurements would not bias the determination of the mean reddening ratio, unless one were to assume that the bluest $(B-V)$ color seen was actually the unreddened standard $(B-V)$ color. Yet no value in the range of intrinsic $(B-V)$ color at maximum assumed in previous analysis, $-0.25 \leq(B-V)_{0}^{\max } \leq-0.04$ has alleviated the mystery of small reddening ratios.

From our analysis, we believe that the cause of the small reddening ratios is the incorrect assumption that there is a unique $(B-V)_{0}^{\max }$. As we have shown here and elsewhere (RPK 96a), the value of $(B-V)_{0}^{\max }$ is actually correlated with the intrinsic luminosity of the SN Ia's. The sense of the correlation is that dimmer SN Ia's have redder intrinsic colors than the brighter and bluer SN Ia's. Separating the color variation intrinsic to the SN Ia's and the color change from dust increases significantly the derived value of the reddening ratios. Under the standard candle assumption, the luminosity variation can be mistaken for absorption, and the correlated color change leads to a bias toward a smaller derived reddening ratio. This bias toward a smaller reddening ratio is demonstrated easily.

In practice, one is trying to measure $A_{V} / E_{B-V}$ by comparing the magnitudes and colors of bright and dim supernovae:

$$
\frac{A_{V}}{E_{B-V}}=\frac{M_{V, \text { dimmer }}-M_{V, \text { brighter }}}{(B-V)_{\text {dimmer }}-(B-V)_{\text {brighter }}},
$$

where the nonzero values of the numerator and denominator are assumed to come from the extinguishing and reddening effects of dust. But what is the value of this ratio in the absence of dust? According to our empirical model of the light and color curves, a supernova that is dimmer by $\Delta$ 
magnitudes in $V$ will be redder by $R_{B-V}(t=0) \Delta$ magnitudes in $(B-V)_{0}$ (see RPK 96a). This gives

$$
\begin{aligned}
\frac{M_{V, \text { dimmer }}-M_{V, \text { brighter }}}{(B-V)_{\text {dimmer }}-(B-V)_{\text {brighter }}} & =\frac{\Delta}{R_{B-V}(t=0) \Delta} \\
& =R_{B-V}(t=0)^{-1}=2.1 .
\end{aligned}
$$

This is manifestly smaller than the canonical galactic value of $\left(A_{V} / E_{B-V}\right)=3.1$. In the presence of dust, any standard candle-based determination of $A_{V} / E_{B-V}$ would be biased toward this low value. Likewise, any absorption corrections based on standard colors and using the canonical value for $A_{V} / E_{B-V}$ would increase the scatter on a Hubble diagram for SN Ia's, as has been seen (Sandage \& Tammann 1993) because the change in color due to $A_{V}$ is not exactly equal to the change in color due to a variation in luminosity.

Van den Bergh (1995) has used the near-coincidence of the reddening vector with the sequence of SN Ia theoretical models of Höflich \& Khokhlov (1996) with various luminosities and colors at maximum to define a corrected luminosity independent of both reddening and supernova model: $M_{V}^{*}(\max )=M_{V}(\max )-3.1(B-V)_{\max }$. Our empirical analysis (RPK 96a) suggests that the luminosity correction of $3.1(B-V)_{\max }$ for dust is not equivalent to the luminosity variation intrinsic to the SN Ia's, $2.1(B-V)_{\max }$. Therefore, it is not surprising to us that when van den Bergh applies his correction to $13 \mathrm{SN}$ Ia's the dispersion increases slightly from $\sigma=0.37$ to $\sigma=0.40$.

\section{CONCLUSIONS}

1. SN Ia intrinsic luminosity and color are related, primarily near maximum. Accounting for this correlation yields optical properties for dust in distant galaxies that are consistent with those of galactic dust. Nevertheless, we cannot rule out the possibility that individual galaxies may have dust whose reddening effects depart from typical galactic absorption.

2. The observed relation between host galaxy absorption for SN Ia's and galaxy morplogy is consistent with the standard picture of the dust content of early- through late-type galaxies.

3. Previous analysis based on the assumption of unique SN Ia luminosity and color at maximum are biased toward lower values for the reddening ratio, $A_{V} / E_{B-V}$, as a result of the inherent correlation between SN Ia intrinsic luminosity and color at maximum. Because of this, attempts to correct for standard absorption can lead to an increase in the distance dispersion. Similarly, a single color-based correction for luminosity and reddening would be ineffective because the change in intrinsic luminosity for a given color difference is not equivalent to the change in observed luminosity for the same color excess.

Understanding the absorbing properties of dust in distant galaxies is of great importance to the measurement of cosmological parameters. Determining the dynamical parameters, $H_{0}$ and $q_{0}$, relies on a comparison of the brightnesses of SN Ia's nearby and further away. A change in either the mean observed extinction or the reddening properties of dust with distance could affect these measurements seriously. For the task of measuring global deceleration from SN Ia's, understanding the properties of dust in the young universe provides a formidable challenge

We are again grateful to Mario Hamuy, Mark Phillips, Nick Suntzeff, and the entire Calán/Tololo collaboration for the opportunity to study their exceptional data before publication. We have benefited from discussions with Peter Garnavich, George Rybicki, and Peter Höflich. This work was supported through NSF grants AST 92-18475 and PHY 95-07695.

\section{REFERENCES}

Bouchet, P Lequeux J Maurice, E, Prevot, L, \& Prevot-Burnichon, M. L. 1985, A\&A, 149, 330

Branch, D., \& Tammann, G. A. 1992, ARA\&A, 30, 359

Brosch, N. 1988, in Dust in the Universe, ed. M. E. Bailey \& D. A. Williams (Cambridge: Cambridge Univ. Press), 502

Brosch, N., \& Loinger, F. 1991, A\&A, 249, 327

Burstein, D., \& Heiles, C. 1982, AJ, 87, 1165

Buser, R. 1978, A\&A, 62, 411

Cadonau, R., Sandage, A., \& Tammann, G. A. 1985, in Lecture Notes in Physics, vol. 224, Supernovae as distance indicators, ed. N. Bartel (New York: Springer), 151

Capaccioli, M., Cappellaro, E., Della Valle, M., D’Onofrio, M., Rosino, L., \& Turatto, M. 1990, ApJ, 350, 110

Della Valle, M., \& Panagia, N. 1992, AJ, 104, 696

Ford, C., et al. 1993, AJ, 106, 3

Hamuy, M., Phillips, M. M., Maza, J., Suntzeff, N. B., Schommer, R. A., \& Aviles, A. 1995, AJ, 109, 1

. 1994, AJ, 108, 2226

Hamuy, M., et al. 1993a, AJ, 106, 2392

Hodge, P. W., \& Kennicutt, R. C. 1982, 87, 264

Höflich, P., \& Khokhlov, A. 1996, ApJ, 457, 500

Iye, M., \& Richter, O. G. 1985, A\&A, 144, 471

Jöeever, M. 1982, Astrofizica, 18, 574
Kron, G. E. \& Mayall, N. U. 1960, AJ, 65, 581

Mathis, J. S. 1990, ARA\&A, 28, 37

Maza, J., Hamuy, M., Phillips, M., Suntzeff, N., \& Aviles, R. 1994, ApJ, 424 L107

Miller, D., \& Branch, D. 1990, AJ, 100, 530

Phillips, M. 1993, ApJ, L105

Riess, A. G., Press, W. H., \& Kirshner, R. P. 1995, ApJ, L17, 438

Riess, A. G., Press, W. H., \& Kirshner, R. P. 1996, in preparation (RPK 96a)

Riess, A. G., et al. 1996b, in preparation

RiFatto, A. 1990, in Dusty Objects in the Universe, ed. E. Bussoletti \&

A. A. Vittone (Netherlands: Kluwer), 277

Sandage, A., \& Tammann, G. A. 1993, ApJ, 415, 1

Sandage, A., et al. 1994, ApJ, 423, L13

1996, ApJ, in press

Savage, B., \& Mathis, J. 1979, ARA\&A, 17

Tammann, G. A. 1987, in IAU Symp. 124, Observational Cosmology, ed. A. Hewitt, G. Burbidge, \& L. Z. Fang (Dordrecht: Reidel), 151 van den Bergh, S. 1995, ApJ, 453, L55

Walterbos, R. 1986, Ph.D thesis, Leiden Univ.

Whittet, D. C. B. 1992, Dust in the Galactic Environment (Cambridge: Cambridge Univ. Press) 